\title{
TWO IDENTITIES FOR LATTICES
}

\section{R. PADMANABHAN ${ }^{1}$}

0. Introduction. In this paper it is shown that every equational class of lattices which can be defined by a finite number of identities can be characterized (among the class of algebras with two binary operations) by means of two identities. In particular, the class of all lattices can be characterized by means of two identities and this solves a problem raised in [11] by Ju. I. Sorkin. In the proof of the main theorem we make use of the known fact that in presence of the lattice axioms the validity of a finite set of lattice identities is equivalent to that of a single one.

1. A binary system $\langle L, *\rangle$ is called a semilattice if the binary operation $*$ is idempotent, commutative and associative. A system $\langle L,+, \cdot\rangle$ is called a lattice if both + and $\cdot$ are semilattice operations in $L$ such that they are connected by the two absorption laws $a+a b$ $=a, a(a+b)=a$.

Lemma. $A$ binary system $\langle L, *\rangle$ satisfying the identities $x * x=x$ and $(x * y) * z=(y * z) * x$ is a semilattice.

This result is proved in [6].

By an equational class of lattices (sometimes a variety of lattices) we mean a class of lattices satisfying certain further identities. Thus distributive lattices and modular lattices form equational classes of lattices. Let $W$ be an equational class of lattices defined by a finite set of identities. By what has been said in the introduction, we may assume that this equational class of lattices is singled out (among the class of all lattices) by a single identity, say

$$
I: f\left(y_{1}, y_{2}, \cdots, y_{n}\right)=g\left(y_{1}, y_{2}, \cdots, y_{n}\right)
$$

where $f$ and $g$ are some words in the variables $y_{1}, y_{2}, \cdots, y_{n}$ and the lattice operations + and $\cdot$. If we want to obtain the equational class of all lattices we need only think of $I$ as the trivial identity $y=y$.

TheOREM. A binary system $\langle L,+, \cdot\rangle$ with two binary operations + and $\cdot$ is a lattice (with + and $\cdot$ as lattice operations) in which the identity $I$ is true if and only if the following two identities hold in it:

Presented to the Society, May 1, 1968; received by the editors June 20, 1967.

1 This work was done while the author had a University Grants Commission Research Fellowship at Madurai University. 


$$
\begin{aligned}
(x f) z+(((a+u) u+v)+w) & =(g z) x+((v+w)+u), \\
x y+y & =y .
\end{aligned}
$$

Proof. The 'only if' part being obvious we need prove only the 'if' part. Let the identities (1) and (2) be true in $\langle L,+, \cdot\rangle$. Put $v=u$ in (1). By (2) we get $(x f) z+(u+w)=(g z) x+((u+w)+u)$. Replacing $z$ by $u+w$ and $x$ by $(u+w)+u$ in the above and applying (2) we get

$$
u+w=(u+w)+u .
$$

Putting $u=x w$ in the above and using (2) we have

$$
w=w+x w .
$$

Now put $w=y u$ in (3). By (4) we have

$$
u=u+u \text {. }
$$

Put $v=w=(a+u) u$ in (1) and apply (2) in the R.H.S. We get, by (5),

$$
(x f) z+(a+u) u=(g z) x+u .
$$

Replacing $z$ by $(a+u) u$ and $x$ by $u$ in the above and using (2) we get

$$
(a+u) u=u \text {. }
$$

By (5) and (6) we have

$$
u u=u .
$$

Now (1) read as

$$
(x f) z+((u+v)+w)=(g z) x+((v+w)+u) .
$$

Putting $z=(u+v)+w$ and $x=(v+w)+u$ in the above we get, by (2),

$$
(u+v)+w=(v+w)+u .
$$

From (5), (8) and the lemma we conclude that + is a semilattice operation in $L$.

Put $u=v=w=(x f) z$ in $\left(1^{\prime}\right)$. We get $(x f) z=(g z) x+(x f) z$.

Similarly, by putting $u=v=w=(g z) x$ in $\left(1^{\prime}\right)$, we have $(x f) z+(g z) x$ $=(g z) x$, and hence by the commutative law for + , we get, for all $x, z, y_{1}, y_{2}, \cdots, y_{n}$ in $L$,

$$
(x f) z=(g z) x .
$$

In particular, putting $y_{1}=y_{2}=\cdots=y_{n}=y$ in the above and using the idempotent laws (5) and (7) we get, for all $x, y, z$ in $L$,

$$
(x y) z=(y z) x .
$$


From (7), (10) and the lemma we conclude that $\cdot$ is also a semilattice operation.

Thus both + and $\cdot$ are semilattice operations and by (2) and (6) we have got both the absorption laws. In other words, the system $\langle L,+, \cdot\rangle$ is a lattice.

Now, putting $x=z=f+g$ in (9) and using the lattice properties of + and $\cdot$, we get $f=g$. Thus the system $\langle L,+, \cdot\rangle$ is a lattice in which the identity $f\left(y_{1}, y_{2}, \cdots, y_{n}\right)=g\left(y_{1}, y_{2}, \cdots, y_{n}\right)$ holds. This completes the proof of the theorem.

CoRollary. The equational class of all lattices, in particular, is defined by the two identities:

$$
\begin{aligned}
(x y) z+(((a+u) u+v)+w) & =(y z) x+((v+w)+u), \\
x y+y & =y .
\end{aligned}
$$

This solves Sorkin's problem.

2. Remarks. The above definition for lattices is simpler than that of Sorkin [11] in every sense of the word. Thus while Sorkin's axiom system contains three identities, one of which is of length twenty-three with nine variables (see p. 49 of [9]), our axiom system contains two identities, the longest identity of which is of length only fourteen with just seven variables.

The above theorem generalizes the results of M. Sholander [10] and M. Kolibiar [4] and provides a uniform method to find a set of two identities defining any equational class of lattices which satisfies the conditions of the theorem; it may, however, be possible to define such a class by two laws in fewer variables or by shorter laws than (1) and (2). Thus, for distributive lattices, for example, (1) is of length nineteen with nine variables. But as proved by M. Sholander in [10], they can be characterized by two identities the largest identity of which is of length only seven with just three variables.

This paper is inspired by a review of [7] and by a similar theorem for groups proved by G. Higman and B. H. Neumann, where it is shown that nearly every variety of groups can be defined by a single identity as a subvariety of groupoids. By contrast, D. H. Potts has shown in [8] that a single law will not suffice to define semilattices. It is not known whether lattices can be characterized by a single identity using only those operations which are derivable from the lattice operations. ${ }^{2}$ A related problem of viewing certain equational classes as part of some other equational classes of a richer algebraic

${ }^{2}$ This was recently solved by R. McKenzie (Added in proof November 20, 1968). 
system and characterizations of the former classes by a single identity in this bigger set up, etc., are treated by G. Grätzer and B. H. Neumann respectively in their forthcoming papers [2] and [5]. Also, we do not know whether there is any equational class of lattices which cannot be defined by a finite set of identities. ${ }^{3}$

I take this opportunity to express my sincere thanks to Professor M. Venkataraman with whom I had stimulating discussions while preparing this paper. My thanks'are also due to Professor G. Grätzer for his interest in this paper.

\section{REFERENCES}

1. G. Grätzer, Review of [7], Math. Rev. 32 (1966), p. 62.

2. - On the spectra of classes of algebras, Proc. Amer. Math. Soc. 18 (1967), 729-735.

3. G. Higman and B. H. Neumann, Groups as groupoids with one law, Publ. Math. Debrecen 2 (1952), 215-221.

4. M. Kolibiar, Lattices in terms of a ternary operation, Czechoslovak Math. J. 6 (1956), 318-329.

5. B. H. Neumann, On a problem of G. Grätzer, Publ. Math. Debrecen (to appear).

6. R. Padmanabhan, On axioms for semi-lattices, Canad. Math. Bull. 9 (1966), 357-358.

7. A. Petcu, Short definitions of lattices, Rev. Roumaine Math. Pures. Appl. 10 (1965), 339-355.

8. D. H. Potts, Axioms for semi-lattices, Canad. Math. Bull. 8 (1965), 519.

9. S. Rudeanu, Axiomele laticilor si ale algebrelor boolene, Bucharest, 1963.

10. M. Sholander, Postulates for distributive lattices, Canad. J. Math. 3 (1951), 28-30.

11. Ju. I. Sorkin, Three-axiom systems for lattices, Ukrain Mat. Ž. 8 (1962), 61-66. (Russian)

MADURAI UNIVERSITY, INDIA AND

UNIVERSITY OF MANITOBA

${ }^{3}$ Kirby Baker and R. McKenzie have given affirmative answers to this question (Added in proof November 20, 1968). 